\title{
Synthesis and Characterization of a series of
}

\section{sulfamethazine multi-component crystals with}

\section{various benzoic acids}

Matthew Scheepers and Andreas Lemmerer

Supplementary Information

Powder X-ray Patterns (PXRD)

$s z+2 c 4 n$

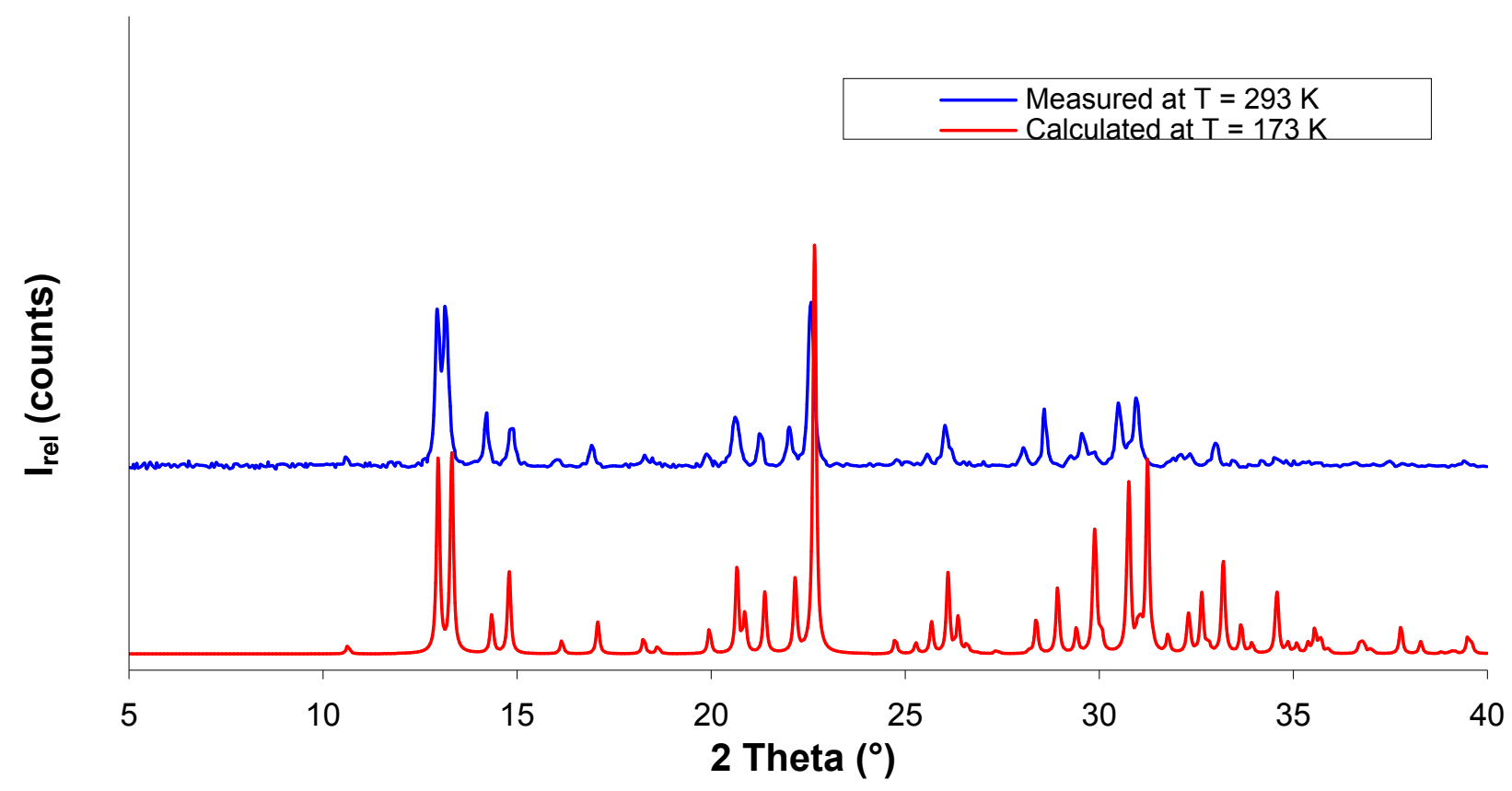

Fig S1 The PXRD pattern for the molecular salt of $\mathrm{sz}+2 \mathrm{c} 4 \mathrm{n}$ showing the calculated and experimentally obtained patterns. 


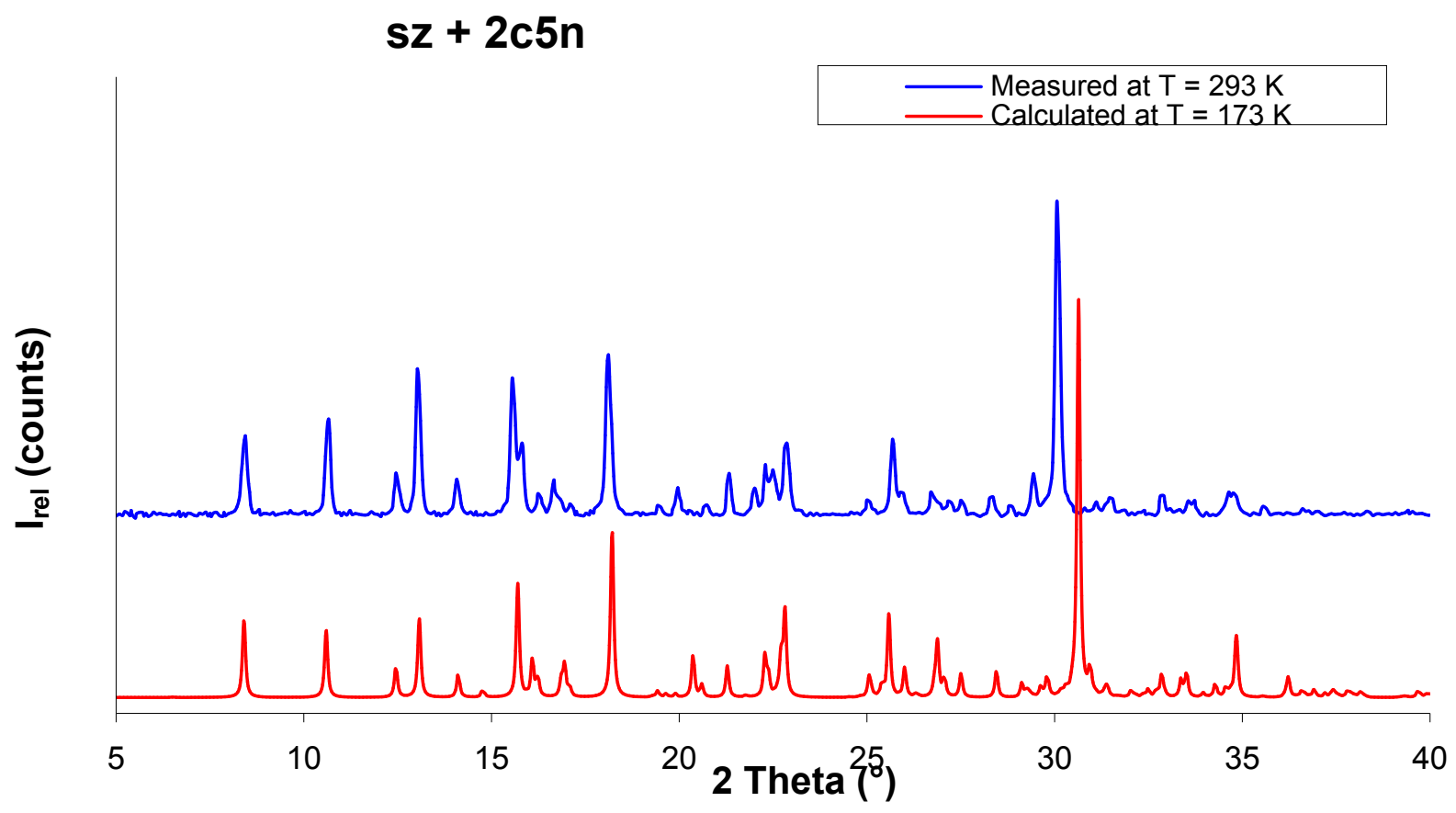

Fig S2 The PXRD pattern for the molecular salt of $\mathrm{sz}+2 \mathrm{c} 5 \mathrm{n}$ showing the calculated and experimentally obtained patterns.

\section{$s z+2 h b a$}

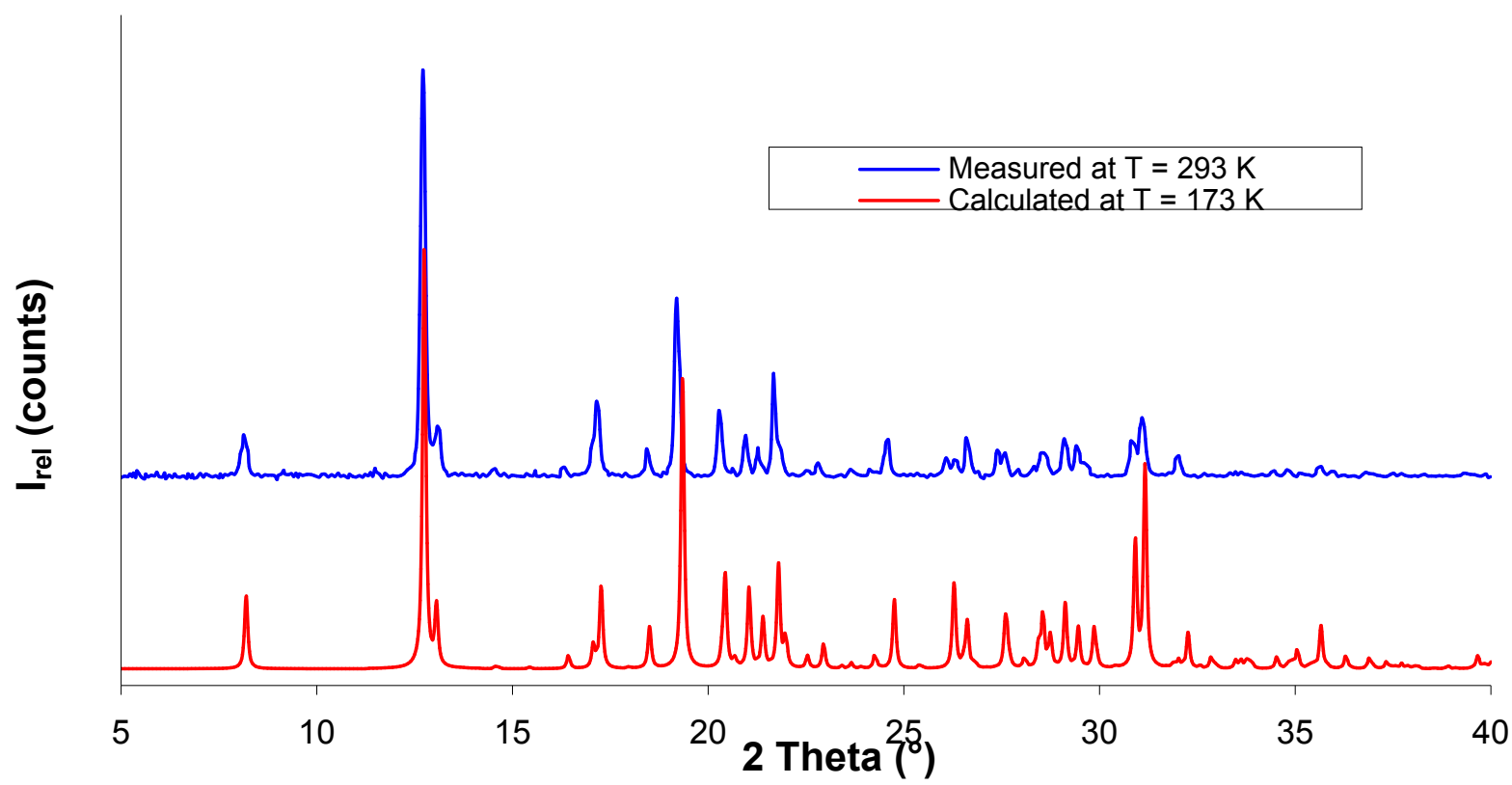

Fig S3 The PXRD pattern for the molecular salt of sz $+2 \mathrm{hba}$ showing the calculated and experimentally obtained patterns. 


\section{$\mathrm{SZ}+3 \mathrm{Hba}$}

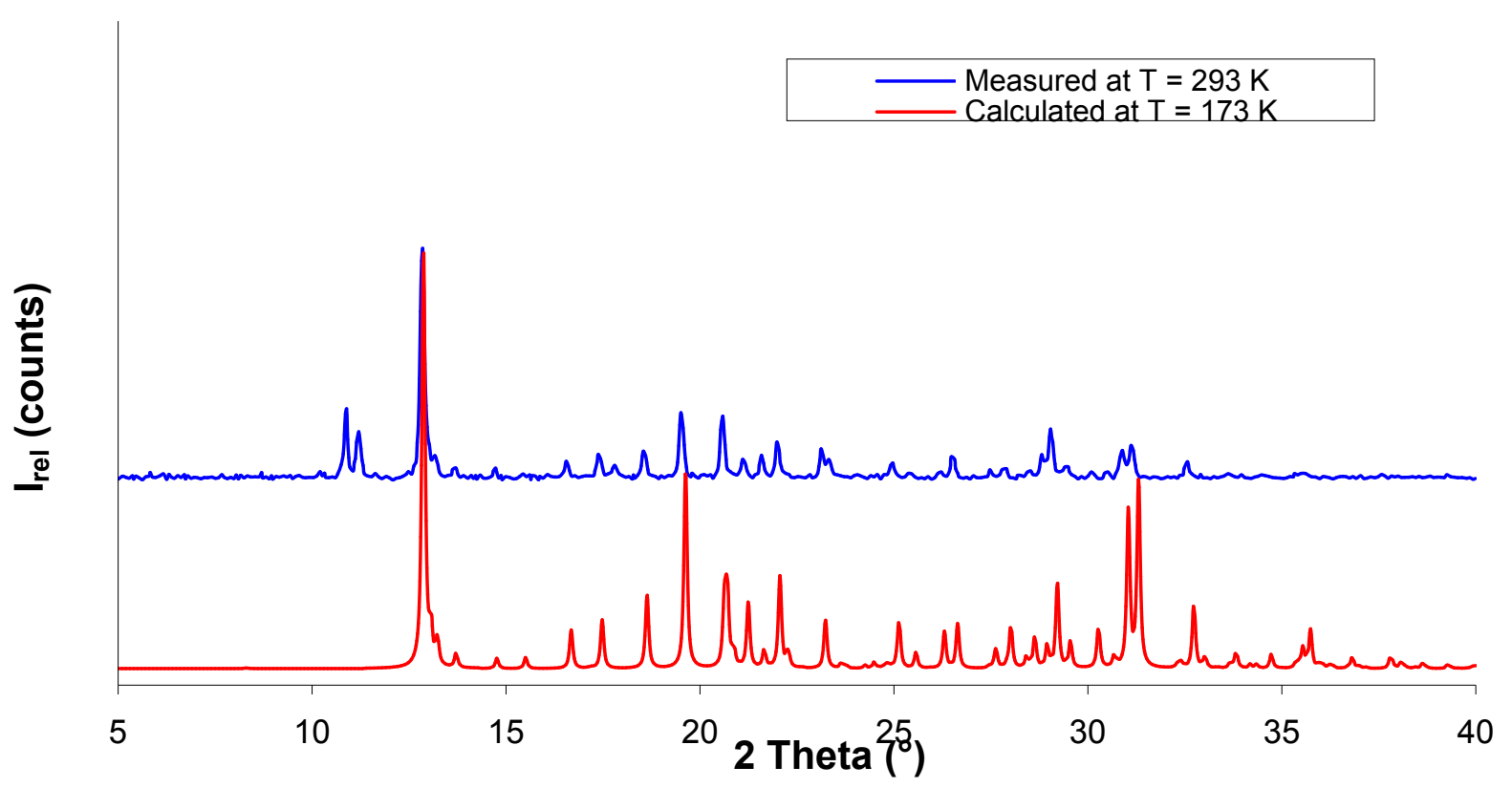

Fig S4 The PXRD pattern for the molecular salt of sz $+3 \mathrm{hba}$ showing the calculated and experimentally obtained patterns.

\section{$s z+4 B r b a$}

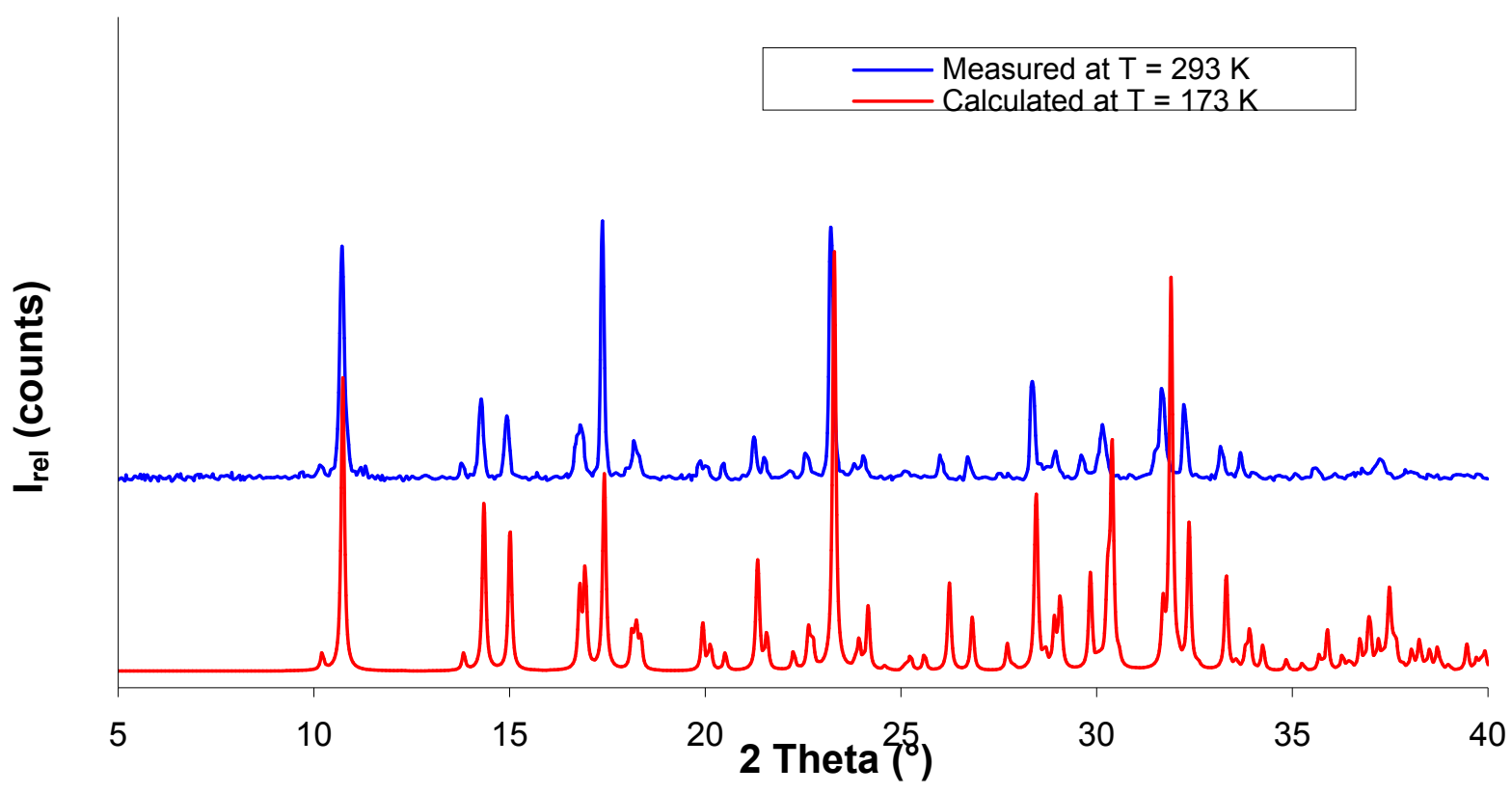

Fig S5 The PXRD pattern for the molecular salt of sz + 4Brba showing the calculated and experimentally obtained patterns. 
sz + 4hba

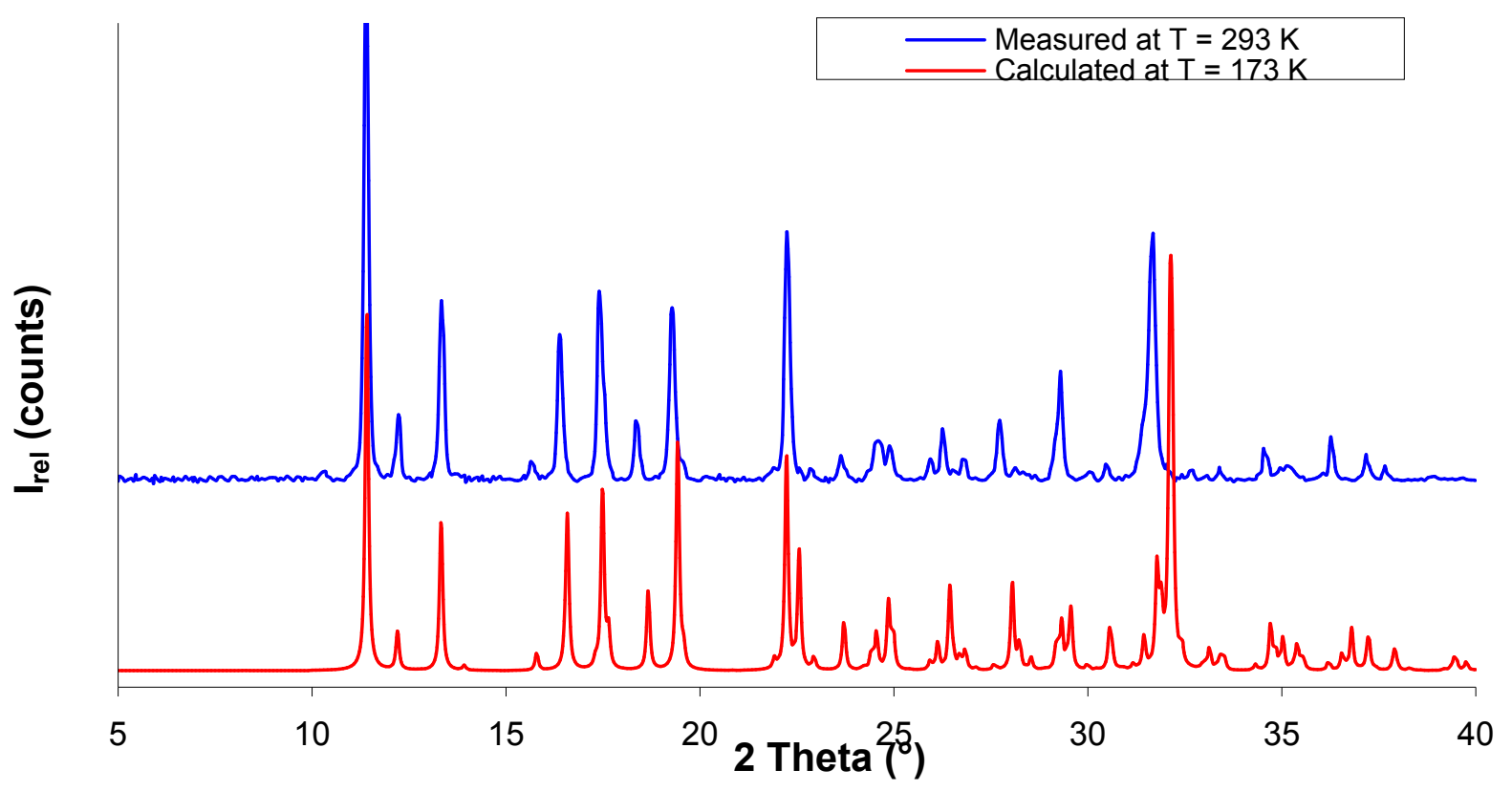

Fig S6 The PXRD pattern for the molecular salt of sz +4 hba showing the calculated and experimentally obtained patterns.

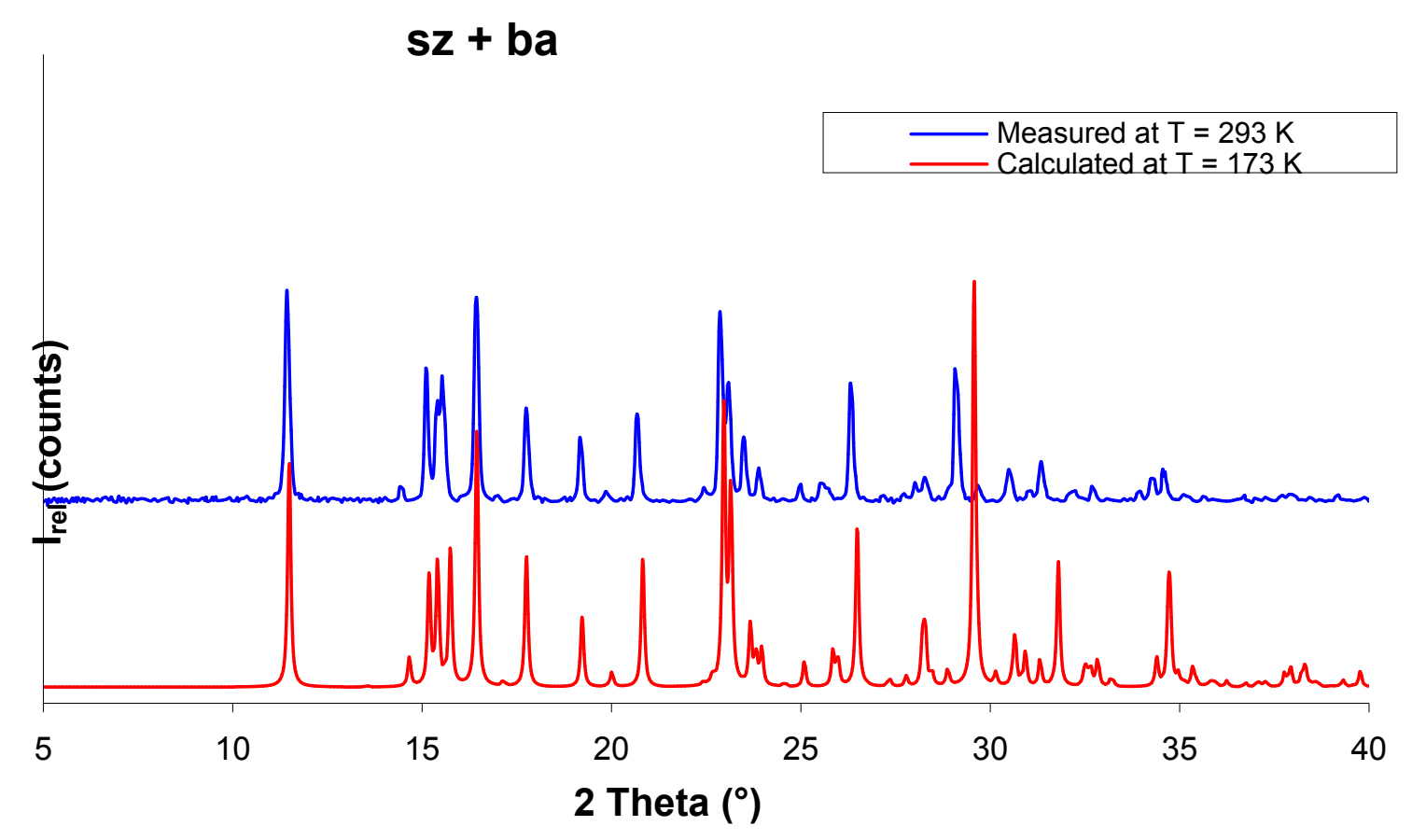

Fig S7 The PXRD pattern for the molecular salt of sz + ba showing the calculated and experimentally obtained patterns. 


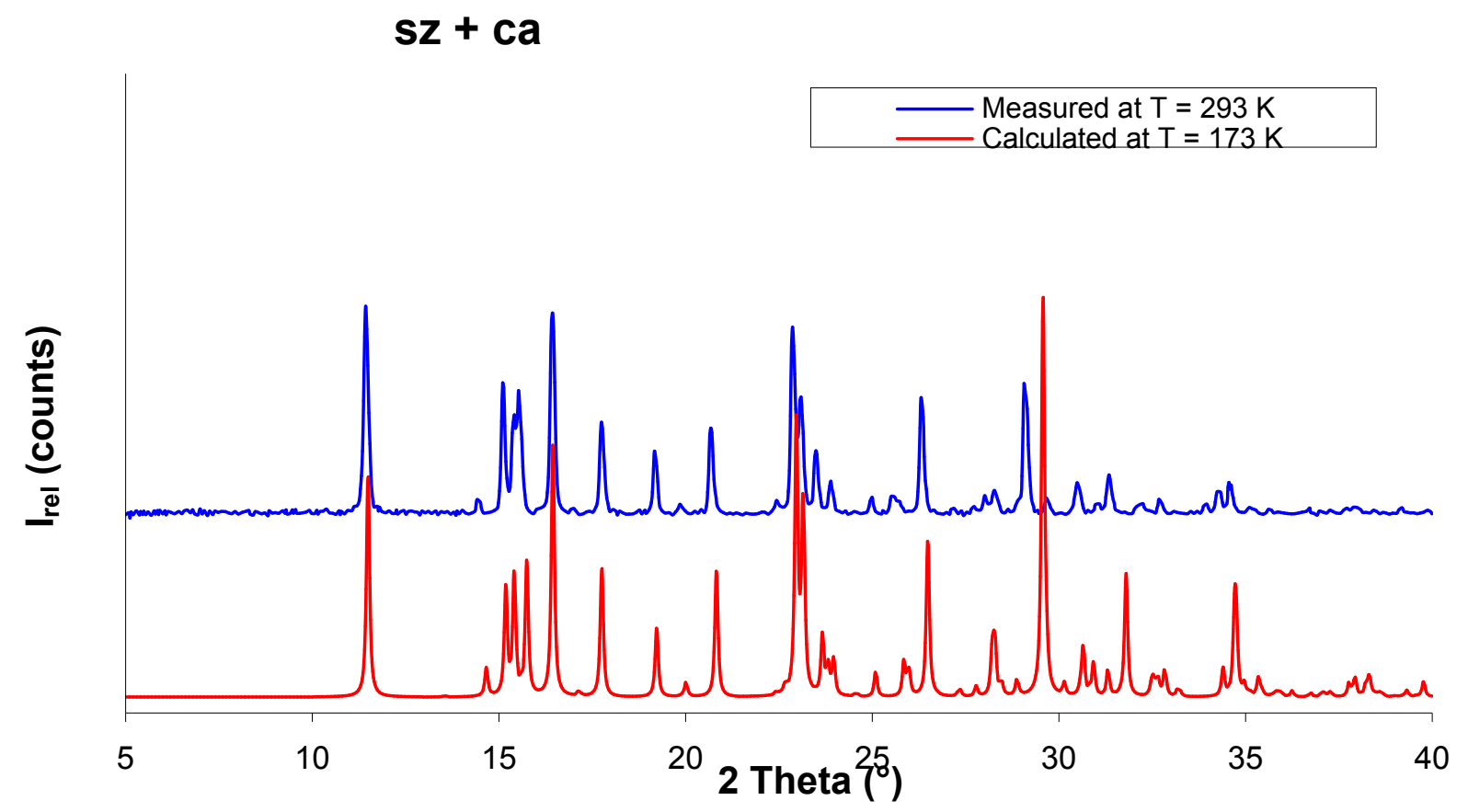

Fig S8 The PXRD pattern for the molecular salt of sz + ca showing the calculated and experimentally obtained patterns.

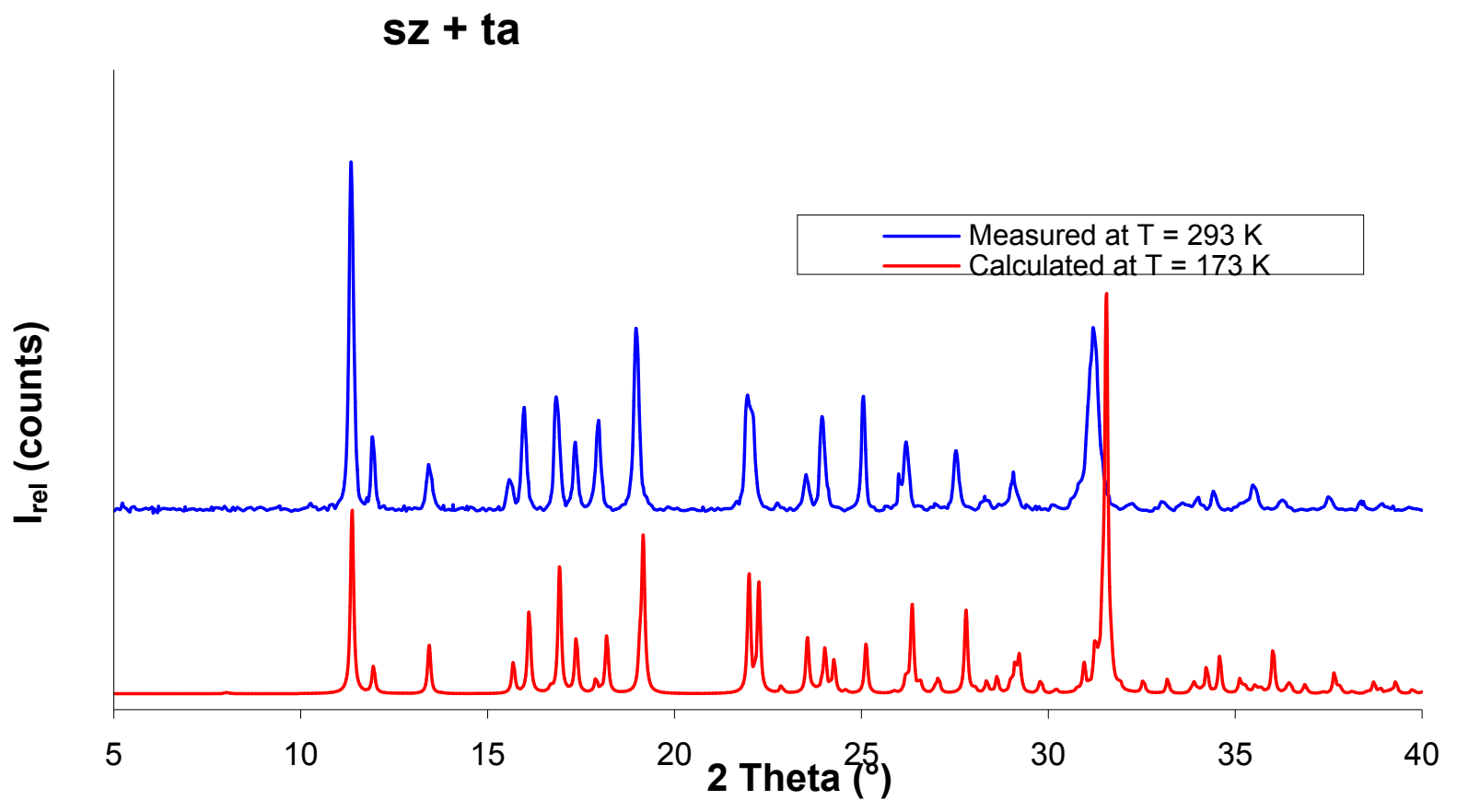

Fig S9 The PXRD pattern for the molecular salt of sz + ta showing the calculated and experimentally obtained patterns. 